Ventura, V. Producción y productividad de la normativa urbana. El trabajo político de los sectores medios en sus contiendas por la ciudad. Derecho y Ciencias Sociales. Mayo- Octubre 2019 NN$^{\circ} 21$. (Derecho, ciudad y propiedad) Pgs 82-103. ISNN 1852-2971. Instituto de Cultura Jurídica y Maestría en Sociología Jurídica. FCJ y S. UNLP

\title{
Producción y productividad de la normativa urbana. El trabajo político de los sectores medios en sus contiendas por la ciudad
}
Production and productivity of urban regulations. The political work of the middle sectors in their disputes over the city

Violeta Ventura ${ }^{\circ}$

\section{Resumen}

La línea Compra de Terreno y Construcción del plan PROCREAR subsidió la demanda de suelo urbano sin regular su oferta. Esto potenció en distintas ciudades del país los límites de la población beneficiara para acceder a dicho bien. Frente a ello les beneficiaries platenses se autoconvocaron solicitando en distintos niveles Estatales la intervención a fin de acceder al suelo urbano acorde a los montos financiados por el Programa de créditos. En menos de un mes el grupo logró incidir en la sanción de una normativa que preveía la rezonificación de tierras rurales en urbanas con destino al PROCREAR así como en sus modalidades de implementación (ordenanza 11.094/13, decreto reglamentario $\mathrm{N}^{\circ}$ 76/14). El artículo se estructura en dos partes. En el primer apartado se busca comprender cómo les beneficiaries (en su mayoría pertenecientes a los sectores medios) se implicaron, movilizaron sus redes e influyeron en el funcionamiento de la estatalidad a fin de lograr su objetivo. Reconstruyendo sus prácticas buscamos iluminar el trabajo político que llevaron adelante para disputar y dar forma a la implementación local de un programa nacional. En un segundo apartado nos detendremos en la productividad urbana y económica de la ordenanza 11.094/13. Analíticamente el caso será abordado, por un lado, desde la perspectiva de la productividad social del conflicto (Melé, 2006); mientras que, por el otro, intentará aportar a la vacancia sobre la relación entre los sectores medios y la producción de ciudad.

Palabras clave: sectores medios, productividad de la normativa urbana, prácticas políticas.

\begin{abstract}
The line "Purchase of Land and Construction" of the PROCREAR plan subsidized the demand for urban land without regulating its offer. This encourages in different cities of the country the limits of the beneficiary population to access that merchandise. Faced with this, the platenses beneficiaries organized themselves requesting the intervention of the Statein order to access the urban land according to the amounts financed by the credit program. In less than a month, the group was able to influence the enactment of a regulation that provided for the rezoning of rural lands in urban areas for PROCREAR as well as their implementation modalities. This article is structured in two parts.

The first section seeks to understand how the beneficiaries (mostly belonging to the middle sectors) get involved, mobilize their networks and influence the functioning of statehood in order to achieve their goal. By rebuilding their practices, we seek to illuminate the political work they carried out to challenge and shape the local implementation of a national program. In a second section we will stop at the urban and economic productivity of

- Licenciada en Sociología por la Universidad Nacional de La Plata (FaHCE-UNLP). Becaria doctoral de CONICET con lugar de trabajo en el Centro de Investigaciones Geográficas del Instituto de Investigación en Humanidades y Ciencias de la Educación (FaHCE-CONICET). violetaventura.1p@gmail.com
\end{abstract}

Recibido: 1/3/2019. Publicable con correcciones: 12/6/2019. 
Ventura, V. Producción y productividad de la normativa urbana. El trabajo político de los sectores medios en sus contiendas por la ciudad. Derecho y Ciencias Sociales. Mayo- Octubre 2019 NN $^{\circ} 1$. (Derecho, ciudad y propiedad) Pgs 82-103. ISNN 1852-2971. Instituto de Cultura Jurídica y Maestría en Sociología Jurídica. FCJ y S. UNLP

ordinance 11094/13. Analytically, the case will be addressed on the one hand from the perspective of the social productivity of the conflict (Melé, 2006); while, on the other, it will try to port the vacancy on the relationship between the middle sectors and the production of the city.

Keywords: middle sectors, productivity of the urban regulations, political practices. 
Ventura, V. Producción y productividad de la normativa urbana. El trabajo político de los sectores medios en sus contiendas por la ciudad. Derecho y Ciencias Sociales. Mayo- Octubre 2019 NN $^{\circ} 1$. (Derecho, ciudad y propiedad) Pgs 82-103. ISNN 1852-2971. Instituto de Cultura Jurídica y Maestría en Sociología Jurídica. FCJ y S. UNLP

\section{Producción y productividad de la normativa urbana. El trabajo político de los sectores medios en sus contiendas por la ciudad ${ }^{1}$}

Violeta Ventura

\section{Introducción}

Uno de los desafíos de la investigación sobre las ciudades latinoamericanas es comprender y visibilizar las diferentes formas de lucha e implicación de las clases medias en las disputas por las formas que asume la urbanización y por la distribución de sus efectos. Como señala Cosacov, "sabemos más sobre cómo los hogares de sectores populares habitan, se apropian, usan la ciudad y movilizan su red de relaciones, que sobre cómo lo hacen las clases medias" (2017a:36). Esta vacancia responde en parte al carácter normalizado de la experiencia urbana de estos sectores (Cosacov, 2017b), a lo que se suma la propia adscripción de clase de les investigadores. Rosaldo (1991) problematizó el detrás de escena de la práctica antropológica señalando la invisibilización de la cultura propia: a mayor poder menor visibilidad cultural; a la inversa, peor es la situación socio-económica de un actor mayor su visibilidad. Sostiene que en el Estado la ciudadanía plena es alcanzada por quienes aparecen como carentes de cultura:

$\mathrm{Si}$ "ellos" tienen un monopolio explícito sobre la cultura auténtica, "nosotros" tenemos una implícita en el poder institucional. Este lado oscuro de la proporción estima la urgencia de reconstruir el análisis social en una forma en que se considere la interacción de cultura y poder, y a la vez hacer que 'nosotros' seamos más visibles culturalmente hablando. (Rosaldo, 1991:4)

En nuestro país autores como Fava (2010), Di Virgilio (2007), Kessler y Di Virgilio (2005 y 2008) y especialmente Cosacov $(2014,2017$ a y 2017b) han visibilizado el hacer normalizado mediante el cual las capas medias intervienen en el espacio urbano.

En este camino abierto, considerando el peso simbólico (Garguín, 2006) y estructural (Adamovsky, 2013) de los sectores medios en la sociedad argentina, el presente artículo aborda el caso de un grupo de "beneficiaries" ${ }^{2}$ del Programa de Crédito Argentino para la Vivienda

\footnotetext{
${ }^{1}$ En este artículo se asume el desafío de hacer un uso inclusivo del lenguaje. Sin embargo se mantendrá el universal masculino en dos ocasiones: cuando refiramos a actores o roles monopolizados por hombres y cuando se citen fragmentos - bibliográficos o de trabajo de campo- que lo hayan utilizado originalmente.

2 Entendemos por "beneficiaries" a aquellas personas que habiéndose anotado en el PROCREAR resultaron ganadoras del sorteo público realizado por Lotería Nacional.
} 
Ventura, V. Producción y productividad de la normativa urbana. El trabajo político de los sectores medios en sus contiendas por la ciudad. Derecho y Ciencias Sociales. Mayo- Octubre 2019 NN $^{\circ} 1$. (Derecho, ciudad y propiedad) Pgs 82-103. ISNN 1852-2971. Instituto de Cultura Jurídica y Maestría en Sociología Jurídica. FCJ y S. UNLP

Única y Familiar (PROCREAR) ${ }^{3}$ quienes organizades en pos del acceso al suelo urbano lograron la sanción de la ordenanza 11.094/13 y su decreto reglamentario $\mathrm{N}^{\mathrm{o}} 76 / 14$. Apostamos con ello a desentrañar las ventajas implícitas que tienen las capas medias para incidir en la vida institucional y en las políticas públicas.

El PROCREAR (2012-2015) estuvo compuesto por distintas líneas. La denominada "Compra de Terreno y Construcción" (CTC) otorgó financiamiento para la construcción de la vivienda y para la compra del terreno donde ésta se emplazaría. La decisión pública de subsidiar a la demanda en mercados de suelo desregulados, potenció procesos especulativos en distintas ciudades del país, evidenciando el error estratégico que significó el lanzamiento de esta modalidad. En la ciudad de La Plata, a los pocos días del primer sorteo de la línea CTC, les beneficiaries comenzaron a experimentar las consecuencias de este desencuentro: ${ }^{4}$ los precios subían, superaban el monto permitido y otorgado por el Banco, y la oferta era escasa. Frente a ello se autoconvocaron solicitando la intervención estatal a fin de acceder al suelo urbano y así aplicar al Programa de créditos. Un mes después el grupo logró incidir en la sanción de una normativa que preveía la rezonificación de tierras rurales en urbanas con destino al PROCREAR, la ordenanza 11.094/13; así como en su decreto reglamentario ( $N^{o}$ 76/14).

Este artículo $^{5}$ se divide en dos apartados. En el primero se busca comprender cómo les beneficiaries -en su mayoría pertenecientes a los sectores medios- se implicaron, movilizaron sus redes, personalizaron la política ${ }^{6}$ e influyeron en el funcionamiento de la estatalidad, logrando la sanción de una normativa urbana. ¿A qué se debe la productividad de sus prácticas? ¿Cuáles fueron sus estrategias para incidir en la decisión pública de generar suelo urbano? ¿Qué lugar ocupó el Estado en esta productividad? ¿Contribuyó, obstaculizó, fue indiferente? Reconstruyendo sus prácticas buscamos iluminar el trabajo político que llevaron adelante para disputar y dar forma a la implementación local de un programa nacional. Detenerse en ello, además, evidenciará el peso de las identidades sociales, la importancia de los capitales desplegados y la selectividad que permeó al Estado en la sanción de la normativa.

\footnotetext{
${ }^{3}$ Se trata de una política -lanzada en 2012 por el Gobierno Nacional- cuyo objetivo habitacional fue la entrega de 400 mil créditos durante los años transcurridos entre 2012 y 2015 y su objetivo macroeconómico dinamizar la economía nacional. Para un análisis del Programa ver Canestraro, 2014; Del Río, 2016; Vértiz, 2016; Ventura, 2015.

${ }^{4}$ Nos referimos al desajuste de una política que subsidiaba la demanda de suelo urbano sin regular su oferta.

${ }^{5}$ Las fuentes principales utilizadas fueron los documentos publicados por los referentes del colectivo en el grupo de facebook "Grupo unificado PRO.CRE.AR LA PLATA terreno + construcción", principal herramienta de comunicación del colectivo. Asimismo se analizaron los intercambios que se daban en el muro de ese mismo perfil y en menor medida entrevistas en profundidad realizadas a miembros del colectivo y a sus representantes. Se realizaron además observaciones participantes durante el proceso de sanción de la ordenanza.

${ }^{6}$ Nos referimos a cómo hacen jugar sus redes para lograr de la estatalidad soluciones personalizadas a diversas necesidades y problemas (Auyero y Benzecry, 2016).
} 
Ventura, V. Producción y productividad de la normativa urbana. El trabajo político de los sectores medios en sus contiendas por la ciudad. Derecho y Ciencias Sociales. Mayo- Octubre 2019 NN $^{\circ} 1$. (Derecho, ciudad y propiedad) Pgs 82-103. ISNN 1852-2971. Instituto de Cultura Jurídica y Maestría en Sociología Jurídica. FCJ y S. UNLP

En un segundo apartado nos detendremos en la productividad urbana y económica de la ordenanza 11.094/13. Los saberes técnicos, las redes de contactos y las oportunidades políticas de les beneficaries, ¿se tradujeron en una capitalización económicamente más allá del propio acceso a la primera propiedad? ¿Quiénes se apropiaron del valor generado por la recualificación del suelo?

Analíticamente el caso será abordado desde la perspectiva de la productividad social del conflicto (Melé, 2006). Coordinando el grupo de investigación Confurb, Melé desarrolló una metodología que, en lugar de analizar las causas de los conflictos urbano-ambientales, se detiene en sus efectos sociales (Azuela y Mussetta, 2009). Desde esta perspectiva la relación entre dinámicas urbanas y sociales se vuelve aprehensible identificando tres áreas de productividad: la territorial, la jurídica y la política (Melé, 2016). ${ }^{7}$ En lo que sigue daremos cuenta de cómo el conflicto en torno al acceso al suelo urbano produjo actores políticos con formas de implicación y negociaciones propias. A su vez, esta participación encontró receptividad en los poderes públicos produciéndose una escena donde -poniéndose en debate la problemática- se la construyó como un problema público (Melé, 2016).

Asimismo, mediante la sanción de una normativa urbana este proceso se materializó en la generación de suelo urbano y de los posteriores barrios. A lo largo del artículo también nos detendremos en la juridificación del conflicto y en la productividad del derecho, el cual, afirma Vértiz (2016), es producto de relaciones sociales y productor de lo social.

\section{Metodología}

Este artículo se sustenta en trabajo de campo realizado durante los años 2013-2016, utilizándose, conforme a los objetivos de la investigación, técnicas propias del enfoque cualitativo.

Se realizaron 17 entrevistas en profundidad a beneficiaries del PRO.CRE.AR. Algunas de ellas (realizadas entre finales de 2013 e inicios de 2014) se centraron en el proceso de sanción de la ordenanza, mientras que otras (2014 y 2016) indagaron en el proceso de su implementación. En los dos casos se empleó un guión semi-estructurado y el muestreo fue selectivo. En el primer grupo, se garantizó contar con las perspectivas tanto de quienes tuvieron una participación activa en la organización colectiva y en la redacción de la normativa, como de les "free rider"

\footnotetext{
${ }^{7}$ En la misma dirección Azuela sostiene que los debates sobre temas urbanos en América Latina se han centrado en torno a si las normativas se cumplen o no, descuidándose "los efectos que sí tienen las normas jurídicas en la organización y en la vida de las ciudades” (Azuela, 2016:10).
} 
Ventura, V. Producción y productividad de la normativa urbana. El trabajo político de los sectores medios en sus contiendas por la ciudad. Derecho y Ciencias Sociales. Mayo- Octubre 2019 NN $^{\circ} 1$. (Derecho, ciudad y propiedad) Pgs 82-103. ISNN 1852-2971. Instituto de Cultura Jurídica y Maestría en Sociología Jurídica. FCJ y S. UNLP

(Olson, 1965). En cuanto al segundo grupo, se priorizaron agentes pertenecientes a los distintos loteos generados por la ordenanza.

Complementariamente se realizaron observaciones durante la gestación y sanción de la ordenanza 11.094/13. Entre los meses de octubre de 2013 y febrero de 2014 se asistió a todas las reuniones y manifestaciones públicas del colectivo, mayormente de regularidad semanal (inclusive mayor hasta la sanción de la norma el 19 de diciembre de 2013). Además, se realizaron observaciones en los distintos loteos generados, algunas durante días corrientes y otras en eventos barriales como fiestas o asambleas. Finalmente, un tercer tipo de observaciones se realizaron en los actos públicos encabezados por funcionarios del Programa o del municipio.

Así mismo, se trabajó sobre los documentos internos generados por les beneficaries, ya sea destinados a la comunicación interna, a la coordinación operativa o a la difusión del colectivo.

Una mención especial ameritan fuentes nodales en esta investigación: los datos, documentos e interacciones generadas en 3 muros de Facebook. ${ }^{8}$ Para analizar el proceso de sanción de la norma y entender las dinámicas y recursos de quienes la impulsaron, se analizaron los muros de los grupos "Grupo Unificado PRO.CRE.AR LA PLATA Terreno + Construcción” (6.874 miembros), "22 de octubre" (14 miembros) y "Condominios Organizados La Plata" (308 miembros). ${ }^{9}$

El uso de las redes sociales en investigaciones sociológicas impone límites metodológicos complejos y (ya no tan) novedosos. En primer lugar, el caudal de información que circula en las redes dificulta su gestión operativa y demanda decisiones en torno a cómo descargarla, guardarla y sistematizarla. En términos analíticos, la interactividad y rapidez que habilita facebook hacen que las interacciones online respondan a lógicas distintas, aunque no escindidas, de las offline. Se decidió trabajar con toda la información publicada en los perfiles seleccionados, es decir no se filtraron datos en su descarga. Todo se sistematizó y analizó (al igual que las fuentes generadas por las entrevistas en profundidad y las observaciones) mediante el software Atlas.ti.

Como contracara las redes sociales como fuentes brindan importantes ventajas. La falta de reactividad y la transcripción inmediata de los datos son dos de ellas. Además, las intervenciones de les beneficiaries mediatizadas por "la pantalla" -es decir, sin tener que

\footnotetext{
${ }^{8}$ Todos ellos son grupos cerrados de Facebook, aunque el "Grupo unificado Pro.Cre.Ar La Plata" funcionó en los hechos como abierto, aceptándose todas las solicitudes que se enviaran.

${ }^{9}$ Los datos corresponden al periodo de escritura de la tesis (inicio de 2019). En todos ellos la cantidad de miembros se mantuvo constante a excepción del grupo Unificado Pro.Cre.Ar La Plata que llego a tener más de 5000 personas a mediados del 2014 y luego siguió creciendo lentamente hasta hoy, sumando más de 1000 miembros.
} 
Ventura, V. Producción y productividad de la normativa urbana. El trabajo político de los sectores medios en sus contiendas por la ciudad. Derecho y Ciencias Sociales. Mayo- Octubre 2019 NN $^{\circ} 1$. (Derecho, ciudad y propiedad) Pgs 82-103. ISNN 1852-2971. Instituto de Cultura Jurídica y Maestría en Sociología Jurídica. FCJ y S. UNLP

enfrentarse cara a cara con sus interlocutores- dan un acceso privilegiado a sus motivaciones, argumentos y sentidos. Finalmente, los registros "en tiempo real" nos permitieron reconstruir el proceso de modo pormenorizado.

\section{El estado no siempre es un laberinto: las clases medias y la productividad de sus prácticas.}

"vos imaginate, un grupo de más de 2.000 personas, tenés biólogos, geólogos, ingenieros, lo que quieras. Entonces decíamos 'necesitamos el plano hídrico' y había una persona que trabajaba obviamente en ADA y conseguías el plano; y teníamos un geofísico, un chico que lo revisó, empezó a ver las cotas ¿entendés? Eso es lo lindo de este grupo también, que decís 'tengo un problema' y tenés un profesional para solucionarlo”. (Fragmento de entrevista a referente del colectivo de beneficiaries del

PROCREAR)

Durante el proceso de implementación del PROCREAR en La Plata, ${ }^{10}$ llamó la atención la rapidez y naturalidad con que les beneficiaries lograron organizarse, realizar gestiones y vincularse a la estatalidad. ${ }^{11}$ Pasadas tres semanas del sorteo y autoconvocades mediante las redes sociales, tuvieron su primera reunión. A partir de ese momento los encuentros fueron frecuentes iniciándose un vertiginoso itinerario que terminó un mes después con la sanción de una normativa urbana impulsada y legitimada por el colectivo. ${ }^{12}$ Durante este mes, además, consolidaron una organización interna eficiente y técnicamente idónea, entablaron un fluido y productivo diálogo con la gestión bruerista ${ }^{13}$ y lograron que se incluyera en la reglamentación de la ordenanza su principal objetivo: elegir la zona donde se localizarían los terrenos y tener control en la gestión de los "vecinos".

La ordenanza 11.094/13 fue aprobada el 19 de diciembre de 2013, incorporándose en su decreto de reglamentación ( $\mathrm{N}^{\mathrm{o}}$ 76/14) tres modos de implementación llamados nativamente Vía 1, Vía 2 y Vía 3. La vía 1 consistió en la apertura de un registro de beneficiaries y otro de

\footnotetext{
${ }^{10}$ La Plata (659.575 habitantes) es una ciudad de peso administrativo y político en la Argentina por ser capital de la provincia de Buenos Aires.

${ }^{11}$ Este artículo se inscribe en el enfoque estratégico-relacional del Estado. En este sentido Jessop (2017) sostiene que el Estado debe ser abordado como ejercicio del poder y como "la condensación mediada institucional y discursivamente (...) de un equilibrio de fuerzas variable y que busca influir en las formas, los propósitos y el contenido de la organización institucional, de la política y de las políticas en coyunturas específicas, marcadas por una mezcla igualmente variable de oportunidades y restricciones, ellas mismas vinculadas a un entorno social más amplio" (Jessop, 2017:97). A ello nos referiremos cuando hablemos de estatalidad.

${ }^{12}$ Mientras en la primera reunión había aproximadamente 50 personas, para el momento de la sanción de la norma el perfil de facebook mediante el cual se organizaban ya contaba con 2.000 integrantes.

13 En el año 2007 Pablo Bruera ganó las elecciones municipales convirtiéndose en intendente de la ciudad. La nueva gestión, de origen vecinalista, llegó a la intendencia luego de 16 años del gobierno de Julio Alak.
} 
Ventura, V. Producción y productividad de la normativa urbana. El trabajo político de los sectores medios en sus contiendas por la ciudad. Derecho y Ciencias Sociales. Mayo- Octubre 2019 NN $^{\circ} 1$. (Derecho, ciudad y propiedad) Pgs 82-103. ISNN 1852-2971. Instituto de Cultura Jurídica y Maestría en Sociología Jurídica. FCJ y S. UNLP

oferentes de tierras. ${ }^{14}$ El vínculo entre las partes sería gestionado por el municipio quien además realizaría las obras necesarias para urbanizar los lotes. Mediante esta vía solo se rezonificó un predio que finalmente fue judicializado. La vía 2 (principal demanda del grupo) permitió que ellas/os mismas/os pudieran promover un loteo. Agrupándose en lo que llamaron "consorcios", debían buscar grandes predios de tierra, entablar las negociaciones con les dueñes y finalmente llevar adelante trámites y obras de rezonificación y subdivisión, todo esto sin mediación estatal. Por esta vía se realizaron 11 rezonificaciones equivalentes a 1.240 terrenos, siendo el mecanismo de mayor peso en la ciudad. La llamada vía 3 extendió el alcance de la ordenanza a favor de quienes -al momento de la sanción de la norma- eran titulares de fracciones indivisas. ${ }^{15}$

Tabla 1. Principales características de las modalidades de aplicación de la ordenanza $11094 / 13$

\begin{tabular}{|c|c|c|c|}
\cline { 2 - 4 } & Vía 1 & Vía 2 & Vía 3 \\
\hline $\begin{array}{c}\text { Promotor del } \\
\text { loteo }\end{array}$ & Municipio & $\begin{array}{c}\text { Beneficiaries del } \\
\text { PROCREAR }\end{array}$ & $\begin{array}{c}\text { Propietaries del } \\
\text { condominio a rezonificarse }\end{array}$ \\
\hline $\begin{array}{c}\text { Origen de la } \\
\text { tierra }\end{array}$ & Privado & Privado & $\begin{array}{c}\text { Miembres de los } \\
\text { condominios }\end{array}$ \\
\hline $\begin{array}{c}\text { Gestión de la } \\
\text { urbanización }\end{array}$ & Municipio & $\begin{array}{c}\text { Beneficiares del } \\
\text { PROCREAR (en la } \\
\text { mayoría de los casos) }\end{array}$ & $\begin{array}{c}\text { Propietaries del } \\
\text { condominio a rezonificarse }\end{array}$ \\
\hline $\begin{array}{c}\text { Financiamiento } \\
\text { de las obras }\end{array}$ & Municipio & $\begin{array}{c}\text { Beneficiares del } \\
\text { PROCREAR (en la } \\
\text { mayoría de los casos) }\end{array}$ & condominio a rezonificarse \\
\hline $\begin{array}{c}\text { Mecanismo de } \\
\text { asignación de } \\
\text { lotes }\end{array}$ & $\begin{array}{c}\text { Dependió del Municipio. } \\
\text { No se explicitó el } \\
\text { mecanismo }\end{array}$ & $\begin{array}{c}\text { Por sorteo. }{ }^{17} \text { Dependió de } \\
\text { les beneficiaries del } \\
\text { PROCREAR }\end{array}$ & Ya eran propietaries \\
\hline
\end{tabular}

Fuente: Elaborada en base al trabajo del equipo de investigación del proyecto H636-SCyT-UNLP.

\footnotetext{
${ }^{14}$ En este caso les propietaries de fracciones rurales debían inscribirse en el Registro de Oferentes administrado por la Municipalidad, presentar una propuesta de subdivisión de los predios y, si cumplían con todos los requisitos, se les atribuía un puntaje en base al cual se determinaba el orden de rezonificación. Posteriormente los nuevos lotes se pondrían a disposición de beneficiarios del PROCREAR, quienes previamente debían anotarse en el Registro de Demandantes.

15 Eran requisitos para ello que el $51 \%$ de sus miembros hubieran sido sorteados en el PROCREAR y que garantizaran -por su cuenta- la provisión de infraestructura básica.

${ }_{16}^{16}$ Adquirieron las tierras antes de la ordenanza.

17 En la mayoría de los casos se realizó un sorteo donde contarían con mayores posibilidades quienes más participación colectiva tuviesen.
} 
Ventura, V. Producción y productividad de la normativa urbana. El trabajo político de los sectores medios en sus contiendas por la ciudad. Derecho y Ciencias Sociales. Mayo- Octubre 2019 NN $^{\circ} 1$. (Derecho, ciudad y propiedad) Pgs 82-103. ISNN 1852-2971. Instituto de Cultura Jurídica y Maestría en Sociología Jurídica. FCJ y S. UNLP

¿A qué respondió la productividad de sus prácticas? La presencia de profesionales y trabajadores estatales en el colectivo fue nodal, dado que posibilitó la traducción casi naturalmente de sus demandas a dispositivos técnicos y lógicas burocráticas. Ya en su primera reunión, por ejemplo, les abogades presentes se ofrecieron a redactar un documento mediante el cual, amparándose en la ley $14.461,{ }^{18}$ se solicitó la intervención estatal ante "los abusos del mercado inmobiliario y los propietarios" (primer documento generado por el colectivo). Citamos este episodio porque permite ver la inmediatez con la que se juridificó el conflicto. Sin embargo, fue durante todo el proceso que el discurso jurídico tuvo un lugar preponderante dentro de sus repertorios: guió decisiones; les permitió participar en la redacción y reglamentación de la norma; una vez sancionada habilitó avanzar en las negociaciones con les dueñes de los predios y con distintos actores de la industria de la construcción, etc.

La traducción, dice Latour (1998), no es otra cosa que una mediación que produce algo nuevo y habilita la articulación entre actores que van conformando durante el proceso nuevas redes. En este sentido la traducción es siempre una instancia de producción de sentido que puede implicar ganancias o pérdidas. A diferencia de otros grupos sociales que para emplazar su problemática en el ámbito del derecho necesitan de especialistas externas/os encargadas/os de traducir jurídicamente sus demandas (Melé, 2016), en el caso que presentamos sus propies protagonistas juridificaron el conflicto quedando en una situación ventajosa. Lo mismo sucedió con quienes tenían otras profesiones afines a la problemática. Hubo ingenieres que asesoraron respecto a la factibilidad de dotar de servicios a distintos predios; agrimensores que proyectaron subdivisiones; arquitectes y contadores que evaluaron ofertas y llevaron adelante gestiones burocráticas/técnicas, etc.

En síntesis, ser sus propias/os "técnicas/os" tuvo un efecto en la re-clasificación jurídica del espacio. La acción mediadora y generalizadora del conocimiento experto y del uso de dispositivos técnicos (registro escrito, certificaciones institucionales, formularios, notas, etc.) permitió también burocratizar sus prácticas (Perelmiter, 2016) agilizando el vínculo con el gobierno municipal.

\footnotetext{
${ }^{18}$ Opera en el documento lo que Melé llama el "efecto del texto", es decir el derecho aparece para los actores de la controversia como un cuerpo de textos posible de ser utilizado como argumentos para fundar sus propios derechos; es decir invocando un texto jurídico los actores esperan un efecto (Melé, 2016). Específicamente esta ley habilita al Estado a transferir inmuebles con destino a PROCREAR. A su vez, de ser necesario realizar subdivisiones para aplicar al Programa, permite que las tierras en cuestión queden exceptuadas del cumplimiento del Decreto-Ley 8.912/77. En concreto en su primera apelación al Estado los pre-adjudicatarios solicitan al municipio que adhiera a la ley 14.461 y proceda "al loteo y venta de tierras fiscales aptas y a precios accesibles; acordes al crédito hipotecario PROCREAR. Se dé trámite expedito en las reparticiones provinciales (Catastro, Registro de la Propiedad Inmueble, etc.) y municipales (Dirección de Obras Particulares, etc.) a las gestiones realizadas para estos créditos; particularmente en lo que a procedimientos de subdivisión de lotes se refiere" (Primer documento generado por el colectivo).
} 
Ventura, V. Producción y productividad de la normativa urbana. El trabajo político de los sectores medios en sus contiendas por la ciudad. Derecho y Ciencias Sociales. Mayo- Octubre 2019 NN $^{\circ} 1$. (Derecho, ciudad y propiedad) Pgs 82-103. ISNN 1852-2971. Instituto de Cultura Jurídica y Maestría en Sociología Jurídica. FCJ y S. UNLP

Además de ser un encuadre para transformarse en interlocutores frente a las autoridades, el saber experto fue una forma hacia adentro del colectivo de producir una diferenciación de roles considerada legítima. Al primer encuentro con el intendente ingresaron quienes estaban "más empapados en las cuestiones legales y técnicas" (documento interno del colectivo). Días después, en base a este subgrupo se conformó la comisión integrada por 8 miembros, la mayoría de ellos profesionales. ${ }^{19}$ Guiadas/os por sus expertises profesionales y un marcado pragmatismo al momento de tomar decisiones, la comisión fue rápidamente legitimada en su rol de representación y conducción del colectivo. Las decisiones respecto a su organización interna también favorecieron la efectividad de sus prácticas. Lo mismo sucedió con las propiedades no dichas de sus trayectorias militantes y profesionales:

“[...] en el trabajo, con mis compañeros, decimos que hacemos Micro Democracia, porque charlamos un montón todo entre nosotros y elaboramos como una propuesta. Cuando está cerradita la presentamos al grupo total y como ya está súper cerrada y prolijita se re vende porque nadie tiene otra [risas] y entonces yo creo que con la comisión un poco pasó eso".

Lo que muestra esta docente universitaria es cómo sus conocimientos prácticos (Bourdieu, 2007) la posicionaron en un lugar privilegiado a la hora de dialogar, negociar y hacer propuestas a la gestión municipal. $^{20}$

Observamos otra ventaja (y acierto) nodal para el grupo. Muches de les beneficiaries pusieron a disposición del resto sus contactos con trabajadores, técnicas/os o funcionarias/os estatales. En la primera semana de vida del colectivo, tomaron contacto con dos senadores, un funcionario de Tierras de Nación, el intendente de la ciudad, ${ }^{21}$ un funcionario de la ANSES y un diputado. Sus redes de relaciones posibles, susceptibles de ser expuestas y movilizadas (Bourdieu, 2007), además de amplias, estaban disponibles para ser activadas. Era el momento de una

\footnotetext{
${ }^{19}$ Una arquitecta, un contador, dos abogados y una estudiante avanzada de abogacía, un ingeniero informático, una antropóloga y un astrónomo.

${ }^{20}$ No queremos sugerir por oposición que los sectores populares no pongan en juego al momento de vincularse a la estatalidad saberes sobre las lógicas de funcionamiento de distintas agencias estatales. De igual modo, desde una perspectiva racionalista, tampoco creemos que dejen de trazar estrategias en sus controversias. Lo que intentamos poner de relieve es que en el modo en que les beneficiaries del PROCREAR vehiculizaron sus demandas, y en parte en la respuesta que obtuvieron, influyó el hecho de que hablaran la misma lengua de la estatalidad.

${ }^{21}$ Siguiendo esta lógica de la movilización de redes interpersonales, una de las beneficiarias consiguió el compromiso de la secretaria del intendente Pablo Bruera de hacerle llegar sus demandas. A los 5 días de su primer encuentro fueron convocados a una reunión con el intendente, realizada el día siguiente.
} 
Ventura, V. Producción y productividad de la normativa urbana. El trabajo político de los sectores medios en sus contiendas por la ciudad. Derecho y Ciencias Sociales. Mayo- Octubre 2019 NN $^{\circ} 1$. (Derecho, ciudad y propiedad) Pgs 82-103. ISNN 1852-2971. Instituto de Cultura Jurídica y Maestría en Sociología Jurídica. FCJ y S. UNLP

capitalización económica del capital social y cultural. ${ }^{22}$ La lógica de movilizar relaciones interpersonales marcó todo el proceso.

Ahora bien, aunque las prácticas de les beneficiares ocuparon un lugar central en la celeridad con la que se logró la sanción de la ordenanza 11.094/13, ésta no dependió exclusivamente de ellas. ${ }^{23}$ Dicha celeridad debe ser leída a contraluz de la espera. Analizando el fenómeno de la dominación política Auyero (2013) encuentra que hacer esperar a los pobres no responde a una debilidad estatal sino a una práctica productiva que reproduce la subordinación política. De este modo, en las interacciones situadas (Perelmiter, 2007) con las burocracias del Estado se crean expectativas en torno a lo que se le puede o no demandar y se performan "las percepciones de los beneficiarios en cuanto a su propio estatus y autoridad en relación con las instituciones y el personal estatal" (Soss citado en Auyero, 2013:23). ${ }^{24}$ Opuesta es la experiencia de les beneficiares del PROCREAR, quienes no contemplaron la espera como algo natural y disputaron un lugar jerarquizado (y profesionalizado) en las tomas de decisiones. En síntesis, en el diálogo que entablaron con el gobierno municipal se sintieron en condiciones de elaborar propuestas y hablar "de igual a igual" con funcionarias/os y técnicas/os.

\subsection{Sentidos y disputas en la modelación de la norma}

Según pudimos reconstruir en el análisis documental, hubo un modelo de ordenanza donde no se contemplaba la posibilidad de que les beneficiaries puedan incidir en la localización y composición de los loteos, puntos centrales en la negociación con la gestión municipal. Frente a ello dos abogades de la comisión ${ }^{25}$ comenzaron a pensar cómo dar a sus objetivos un marco legal, iniciándose un diálogo entre las/os técnicas/os del colectivo y los del municipio.

En pocas ocasiones el colectivo tensó su vínculo con el bruerismo, una de ellas fue cuando éste quiso excluir la vía 2 de la norma. ${ }^{26}$ Sostenemos que las motivaciones que guiaron las prácticas

\footnotetext{
${ }^{22}$ Avanzaremos sobre este punto en el segundo apartado.

${ }^{23}$ La gestión bruerista ocupó un lugar central mediante una efectiva canalización de esta demanda. Sin embargo, este artículo no se detendrá en el escenario político local por una cuestión de extensión. También es relevante para pensar la celeridad del proceso el lugar que en la organización colectiva tuvo la red Facebook, habilitando un dinamismo particular.

${ }^{24}$ En síntesis, para Auyero la espera forma parte de una estrategia exitosa de dominación política cuyo "principal efecto positivo es la creación cotidiana de sujetos que saben que cuando interactúan con las burocracias estatales tienen que cumplir pacientemente con los requisitos arbitrarios, ambiguos y siempre cambiantes que impone el Estado y que actúan en consecuencia" (Auyero, 2013:24).

${ }^{25}$ Esto se complementó en la conformación de un grupo informal de abogades que por fuera de la comisión discutía técnicamente las problemáticas.

${ }^{26}$ La negativa del municipio a su incorporación estaba basaba en los costos políticos que esto pudiera tener, específicamente que se generen cuestionamientos por favorecer el crecimiento disperso y el negocio inmobiliario. Fue importante en esta negociación el hecho de que el 23 de noviembre, en su segunda reunión como colectivo, les beneficiaries decidieron comenzar a buscar fracciones indivisas en vistas a posibles rezonificaciones. Toda la información obtenida en este relevamiento fue sistematizada en un sofisticado formulario on-line diseñado por elles mismes, para que luego les profesionales del colectivo evaluaran la conveniencia de cada oferta de terrenos.
} 
Ventura, V. Producción y productividad de la normativa urbana. El trabajo político de los sectores medios en sus contiendas por la ciudad. Derecho y Ciencias Sociales. Mayo- Octubre 2019 NN $^{\circ} 1$. (Derecho, ciudad y propiedad) Pgs 82-103. ISNN 1852-2971. Instituto de Cultura Jurídica y Maestría en Sociología Jurídica. FCJ y S. UNLP

de les beneficiares en esta contienda están vinculadas a sus sentidos en torno al lugar que ocupan en el espacio social; y que, además, incidieron en la producción de ciudad. En un documento interno del colectivo, previo a la ordenanza, la comisión sistematizó las ventajas y desventajas de la modalidad que impulsaban:

\begin{tabular}{|c|c|}
\hline Ventajas & Desventajas \\
\hline $\begin{array}{l}\text { "Elección más libre de los lotes, } \\
\text { trato directo con vendedores y la } \\
\text { posibilidad de tener herramientas } \\
\text { de negociación que permita } \\
\text { obtener mejores beneficios, } \\
\text { sapienza de quienes van a } \\
\text { conformar el futuro barrio y la } \\
\text { experiencia de trabajar en } \\
\text { conjunto." }\end{array}$ & $\begin{array}{l}\text { "Gran parte de los trámites para el } \\
\text { loteo deben hacerse por parte de les } \\
\text { beneficiaries previos a la división } \\
\text { (salvo que esté negociado } \\
\text { oportunamente con el propietario) lo } \\
\text { que demanda tiempo, tal vez sea } \\
\text { necesario erogar algún monto en } \\
\text { concepto de seña." }\end{array}$ \\
\hline
\end{tabular}

A pesar de tener que invertir más tiempo y dinero, les beneficiaries pelearon por esta modalidad. Sus expectativas en torno al habitar de los futuros barrios ocuparon un lugar central en esta decisión, por la posibilidad de incidir en la localización o en la composición de los loteos. La búsqueda de socialización entre iguales reprodujo un modo auto-segregado de producir ciudad.

Por otra parte, en esta fuente se explicita algo que operó durante todo el proceso: sus prácticas se guiaron por la convicción de que sus gestiones serían más eficientes que las del Estado municipal. Esta auto-jerarquización muestra la configuración diferenciada -respecto a otros sectores sociales- de expectativas sobre lo que podían pedir (y lo que no) al Estado, así como cuáles eran las respuestas esperables. Cuenta una entrevistada que, al momento de realizar el proyecto hidráulico, el municipio restringió la cantidad de ingenieros habilitados para hacerlo. Sostenía además que estos profesionales habían pagado coima a los funcionarios municipales para monopolizar la práctica. Les beneficiaries no aceptaron los servicios argumentando que les

El 30 de noviembre realizaron la tercera reunión del colectivo y en base a las 500 hectáreas relevadas generaron subgrupos de trabajo por zonas de la ciudad. 
Ventura, V. Producción y productividad de la normativa urbana. El trabajo político de los sectores medios en sus contiendas por la ciudad. Derecho y Ciencias Sociales. Mayo- Octubre 2019 NN $^{\circ} 1$. (Derecho, ciudad y propiedad) Pgs 82-103. ISNN 1852-2971. Instituto de Cultura Jurídica y Maestría en Sociología Jurídica. FCJ y S. UNLP

profesionales que ellos podían aportar, eran significativamente mejores que "los cuatro de copas" que proponía el municipio:

"nosotros igual fuimos con otro, que conocía otra chica del loteo, que es el director de la Universidad Tecnológica Nacional, entonces no lo podían cuestionar, porque éstos eran unos cuatro de copas y nosotros fuimos con el mejor...".

Por su parte, la incorporación de la vía 3 a la norma ilumina el lugar de la circunstancialidad política en la implementación local de una política nacional. Un día antes de que se sancione la ordenanza, un funcionario municipal de segunda línea -propietario de un terreno en condominio- convocó a miembros de distintos condominios, informándoles la posible incorporación de un artículo que habilitaría el cambio de uso de suelo de sus tierras. Surgía así la red de Condominios Organizados de La Plata ${ }^{27}$ (COLP), un grupo independiente al colectivo que transformó en pública la problemática (les impulsores de la vía 2). Les propietaries de terrenos en condominio leyeron esa convocatoria como una oportunidad política inédita y difícil de repetir.

"Desde antes sabíamos que presionar por la rezonificación iba a ser una cuestión política, más allá de lo técnico, la coyuntura general aportó para que nosotros pudiéramos ser la vía 3 [...]. Dijimos 'chicos está ésta posibilidad, la venimos remando hace un montón de años, vamos a juntarnos a ver si nos podemos meter por la ventana' y bueno, nos pudimos meter por la ventana”.

Ahora bien, además de la oportunidad, les beneficaries pudieron posicionarse frente a ella aventajadamente, accediendo a recursos estatales mediante la personalización de la política:

"...muchos condominios tuvieron sus representantes que fueron los que llevaron adelante las gestiones, con la particularidad de que es mucha gente que trabaja en el Estado, entonces, además de tener voluntad, conoce los laberintos del Estado, sabe por

\footnotetext{
${ }^{27}$ Dice un entrevistado: “A mediados del mes de diciembre del 2013, fuimos convocados a una reunión de manera informal, en donde se nos comentó sobre la voluntad del ejecutivo municipal de lograr la creación de nuevos lotes [...] En ese momento nacía Condominios Organizados La Plata (COLP), un espacio donde todos los propietarios de tierras indivisas con planes PROCREAR, teníamos la posibilidad de luchar en forma conjunta por el sueño de la casa propia".
} 
Ventura, V. Producción y productividad de la normativa urbana. El trabajo político de los sectores medios en sus contiendas por la ciudad. Derecho y Ciencias Sociales. Mayo- Octubre 2019 NN $^{\circ} 1$. (Derecho, ciudad y propiedad) Pgs 82-103. ISNN 1852-2971. Instituto de Cultura Jurídica y Maestría en Sociología Jurídica. FCJ y S. UNLP

dónde tiene que ir, cómo se tiene que mover, donde están los expedientes. Por ahí si hubiésemos sido gente que no está en la burocracia hubiéramos pasado de largo."28

Ser nativas/os de los laberintos del Estado (además de sus arquitectes, abogades, ingenieres, burócratas, etc.) hizo posible orientar la forma que asumió la implantación local del PROCREAR. Nuevamente, como en la tecnificación de sus demandas, la eficacia en conseguir los recursos dependió de sus propias expertise y relaciones. Elles mismes resultaron efectivas/os, no requirieron de un/a mediador/a para "acelerar" el tiempo de espera en una agencia estatal, ni para saber cómo o dónde presentar un escrito, como sí suele ser el caso en los sectores populares (Auyero y Benzecry, 2016). Elles mismes son portadores de capitales y movilizan su conocimiento y sus redes para que una persona conocida les haga un favor.

En este artículo proponemos que -al igual que en la red clientelar- hay dentro de los sectores medios una personalización de la política para conseguir recursos, apareciendo la política en su dimensión instrumental. Sin embargo, a diferencia de la red clientelar que depende de agentes mediadores (punteros) que operan como núcleos de distribución de recursos y protección contra los peligros de la vida diaria (Auyero y Benzecry, 2016), no se encuentra esa figura de mediación en el caso de las clases medias. Esta personalización de la política "por mano propia" da cuenta de una relación más simétrica con la estatalidad $\mathrm{y}$, en particular, con las autoridades municipales. Ese es un elemento que coloca en un lugar diferente esta red de favores respecto de la red clientelar: no es una "amistad desnivelada" (Auyero y Benzecry, 2016) ni una relación de dominación sustentada en la "inequidad"; al contrario, esta red de favores es entre iguales, cuidadosamente administrada y dosificada.

Llegado este punto es necesario explicitar una consideración analítica que subyace a lo sostenido anteriormente. Hay una tendencia en la literatura sociológica clásica a pensar el Estado desde sus "dificultades" para cumplir funciones sistémicas; regular las relaciones sociales; conformar burocracias fuertes técnica y administrativamente y sobre todo autónomas respecto a los distintos gobiernos y partidos políticos. La "tesis de la debilidad del Estado argentino" (Perelmiter, 2007) tuvo, y aun conserva, un peso importante en nuestras ciencias sociales; por ello, asegura Perelmiter, no hay que detenerse en evaluar la fortaleza o debilidad del Estado, sino en "los entramados micro sociales entre actores, prácticas y relaciones que hacen al funcionamiento cotidiano de aquello que llamamos Estado" (Perelmiter, 2007:1). Siguiendo las prácticas de les beneficiaries del PROCREAR (el modo en que se relacionan entre sí y con los distintos agentes del Estado y la forma en que movilizaron sus redes

\footnotetext{
${ }^{28}$ La bastardilla es nuestra.
} 
Ventura, V. Producción y productividad de la normativa urbana. El trabajo político de los sectores medios en sus contiendas por la ciudad. Derecho y Ciencias Sociales. Mayo- Octubre 2019 NN $^{\circ} 1$. (Derecho, ciudad y propiedad) Pgs 82-103. ISNN 1852-2971. Instituto de Cultura Jurídica y Maestría en Sociología Jurídica. FCJ y S. UNLP

profesionales e interpersonales) buscamos mostrar algunas de las racionalidades que se enlazaron en el micro escenario municipal y que operaron en la gestión de la falta de suelo urbano. Asimismo, a la luz del lugar de privilegio que en nuestras sociedades ocupa la informalidad en la circulación y ejercicio del poder (Perelmiter, 2007), buscamos reconstruir la productividad política de estas interacciones situadas entre funcionarios, técnicos y beneficiaries en la implementación local de una política contra-cíclica nacional.

\section{La capitalización económica de oportunidades, redes y saberes}

Al mismo tiempo que generó condiciones de inclusión social gracias a la generación de empleo, el PROCREAR atendió las necesidades habitacionales de un sector que estaba excluido del sistema de crédito (Del Río, 2017). Además, estuvo fuertemente subsidiado por el gobierno nacional mediante tasas de interés fijas y bajas (entre 2 y $18 \%$ ) así como capital invariable. En economías inflacionarias como la argentina, esta cualidad es especialmente conveniente para les tomadores de deuda. Por otra parte, el Programa (y las prácticas de les beneficiares) se inscriben en un proceso de inquilinización que tuvo lugar en Argentina y particularmente en la ciudad durante el periodo intercensal 2001-2010. ${ }^{29}$

A la luz de estas cualidades entendemos al PROCREAR como una política progresista que favoreció la distribución secundaria de ingresos (Danani, 2009). ${ }^{30}$ No obstante ello en La Plata, tras el lanzamiento de la línea CTC, el arreglo urbanístico generado no logró quebrar -incluso acompañó- la inercia de la producción desigual y difusa de ciudad. ${ }^{31}$

Como señalamos en el apartado anterior, en sus interacciones con el gobierno municipal y con distintitas agencias estatales, les beneficiaries lograron el cambio de usos de suelo de rural (intensivo, extensivo o complementario) a urbano. Estas rezonificaciones produjeron una fuerte valorización en su precio: el derecho no solo es producto de relaciones sociales sino también productor de lo social (Vértiz, 2016). La capacidad de les beneficaries de incidir en la estatalidad, trascendió el acceso individual al suelo urbano: ¿con qué modos de producción de

\footnotetext{
${ }^{29}$ La población platense vivió un fuerte proceso de inquilinización conforme a los cambios que se dieron en el Régimen de Tenencia de la Vivienda. Durante el periodo mencionado la cantidad de inquilinos creció un 5,7\% mientras que la cantidad de propietarios del terreno y la vivienda descendió un 5,8\%.

${ }^{30}$ Que por definición solo existe por la mediación estatal.

${ }^{31}$ La ordenanza 11.094/13 no fue la única opción que se manejó para la ciudad. Tres meses después de su sanción la ANSES creó la Dirección de Desarrollo de Suelo Urbano (DDSU) de PROCREAR orientada a mitigar, mediante la generación de "Lotes Con Servicios", los desajustes generados por el lanzamiento de la línea CTC. Para la ciudad de La Plata la DDSU promovió 628 parcelas, 92 en tierras nacionales y 536 en tierras privadas. Recién el 13 junio de 2017 se realizó el sorteo por Lotería Nacional de los 536 lotes generados en la localidad de San Carlos, tres años después de la sanción de la ordenanza y dos desde el triunfo en las elecciones presidenciales de la alianza Cambiemos, gestión que relanzó el PROCREAR con importantes modificaciones. La respuesta del gobierno nacional, entonces, fue extemporánea y no absorbió la demanda de les beneficiaries. Además preveía menos de la mitad de parcelas que la ordenanza 11.094/13, la cual produjo 1.240 lotes mediante la vía 2, 247 por la vía 1 y una cantidad similar mediante la vía 3 (Del Río, 2017).
} 
Ventura, V. Producción y productividad de la normativa urbana. El trabajo político de los sectores medios en sus contiendas por la ciudad. Derecho y Ciencias Sociales. Mayo- Octubre 2019 № 21. (Derecho, ciudad y propiedad) Pgs 82-103. ISNN 1852-2971. Instituto de Cultura Jurídica y Maestría en Sociología Jurídica. FCJ y S. UNLP

ciudad dialogó la ordenanza 11.094/13? ¿Quiénes se apropiaron del valor generado por la recualificación del suelo?

A diferencia de aquellos municipios donde se movilizaron tierras fiscales e hicieron acuerdos de financiamiento para su urbanización, en La Plata con la ordenanza 11.094/13 la demanda de suelo se gestionó en torno a otros dos elementos (Del Río, 2017). El primero de ellos fue la convalidación del mercado como timonel del proceso, siendo la vía 2 un contrato entre privados donde el Estado no intervino regulando el precio del suelo. El segundo fue "la apertura indiscriminada del perímetro urbano, lo cual generó y reforzó una configuración urbana más extensa y difusa, con el consabido costo ambiental, social y económico que esto produce en términos colectivos" (Del Río, 2017:298). ${ }^{32}$

En este sentido, Del Río asegura que más allá de la productividad del proceso organizativo frente a la falta de previsión estatal, no puede considerarse un proceso pleno de autogestión ya que no se interpelaron las relaciones de poder del orden urbano. Tampoco podemos considerarlo un proceso democratizador y redistributivo ya que las prácticas de les beneficiares -en confluencia con la decisión política del municipio- contribuyeron a "significativas transferencias de renta a los propietarios de la tierra y promovieron un horizonte fragmentado en el modo de procesar el crecimiento urbano a nivel municipal.” (Del Río, 2017:301).

En el discurso de les beneficiaries que impulsaron la vía 2 subyace una idea al respecto: con la presión social que ejercieron, lograron aumentar su capacidad de negociación y acceder al suelo a precios más bajos que los ofrecidos por el mercado. Sin embargo, sostiene Vértiz (2016), esta idea es cierta solo en parte. Dando cuenta de los efectos económicos de la normativa Vértiz calcula la renta inducida por las rezonificaciones y subdivisiones de las vías 2 y 3 , así como la plusvalía "transferida a los propietarios del suelo y la magnitud de la misma pudo ser apropiada por los usuarios-beneficiarios del programa" (Vértiz, 2016:161).

\footnotetext{
${ }^{32}$ En resumen, el municipio se limitó a ocupar un rol de facilitador frente a "la propuesta de los beneficiarios, quienes desde una lógica pragmática y voluntarista propusieron compra de tierra indivisa, urbanizarla $\mathrm{y}$ subdividirla en función de su necesidad.” (Del Río, 2017: 298).
} 
Ventura, V. Producción y productividad de la normativa urbana. El trabajo político de los sectores medios en sus contiendas por la ciudad. Derecho y Ciencias Sociales. Mayo- Octubre 2019 NN $^{\circ} 1$. (Derecho, ciudad y propiedad) Pgs 82-103. ISNN 1852-2971. Instituto de Cultura Jurídica y Maestría en Sociología Jurídica. FCJ y S. UNLP

Tabla 2. Características de los loteos generados por las vías 2 y 3 y precios de referencia de la tierra.

\begin{tabular}{|c|c|c|}
\hline & Vía 2 & Vía 3 \\
\hline Loteos generados & 11 & 14 \\
\hline Lotes generados & 1.240 & Sin datos \\
\hline $\begin{array}{l}\text { Superficie promedio } \\
\text { de los lotes }\end{array}$ & $399 \mathrm{~m}^{2}$ & $1.080 \mathrm{~m}^{2}$ \\
\hline $\begin{array}{c}\text { Precio promedio de lotes con } \\
\text { servicios }\end{array}$ & $349 \$$ por $\mathrm{m}^{2}$ & $75 \$$ por $\mathrm{m}^{2}$ \\
\hline $\begin{array}{l}\text { Precio promedio de tierra bruta } \\
\text { mayor a los } 10.000 \mathrm{~m} 2 \text {. }\end{array}$ & $60 \$$ por $\mathrm{m}^{2}$ & $30 \$$ por $\mathrm{m}^{2}$ \\
\hline $\begin{array}{l}\text { Precio promedio de terrenos } \\
\text { subdivididos. Entre } 250 \text { y } 1500 \text { m2. }\end{array}$ & $547 \$$ por $\mathrm{m}^{2}$ & $547 \$$ por $\mathrm{m}^{2}$ \\
\hline
\end{tabular}

Fuente: Elaborado en base a Vértiz, $2016 .^{33}$

Los lotes con servicios generados mediante la vía 2 tuvieron un precio promedio de $349 \$ / \mathrm{m}^{2}, 34$ pagando por el lote final un $25 \%$ menos de lo que hubiesen pagado por suelo urbano en las mismas localizaciones recurriendo individualmente al mercado. ${ }^{35}$ Como contracara, los propietarios originales de la tierra "se apropiaron de una renta de $115 \$ / \mathrm{m}^{2}$ equivalente a una ganancia extraordinaria del 192\% en un plazo de poco más de un año" (Vértiz, 2016:172). ${ }^{36}$ Fueron justamente les oferentes de un mercado sin regulación quienes capturaron la mayor parte de la valorización producida por la decisión pública de recualificar suelo.

En el caso de la vía 3 se produjo un aumento del 650\% del valor de las tierras. En este caso la valorización inducida por la normativa fue capturada por les beneficiares del PROCREAR. Habiendo comprado las tierras antes de la sanción de la norma (incluso antes del lanzamiento de la línea CTC), les propietaries de terrenos en condominio se anticiparon a la suba del precio de suelo inducida por esta decisión estatal. ${ }^{37}$

\footnotetext{
${ }^{33}$ Para precisiones metodológicas sobre el cálculo de la renta inferida por el cambio de zonificación, ver Vértiz (2016).

${ }^{34}$ De los cuales un 34\% corresponde al costo de urbanización de los mismos, asumido por los beneficiaros, en ese entonces futuros usuarios de las tierras. Para el caso de la vía 3 el costo de urbanización asciende a un 50\%.

${ }^{35}$ En esas mismas zonas el $\mathrm{m}^{2}$ de los terrenos de entre 250 y $1.500 \mathrm{~m}^{2}$ valía 547 pesos (Vértiz, 2016).

${ }^{36}$ Los beneficiarios pagaron $349 \$ / \mathrm{m}^{2}$ mientras que el precio promedio de tierra bruta mayor a $10.000 \mathrm{~m}^{2}$ para el área rural o complementaria era de $60 \$ / \mathrm{m}^{2}$.

${ }^{37}$ En términos concretos, mediante la vía 3 les les propietaries de terrenos en condominio lograron apropiarse de una renta de $475 \$ / \mathrm{m}^{2}$, pagando $72 \$ / \mathrm{m}^{2}$ frente a los $547 \$ / \mathrm{m}^{2}$ por los que se ofertaban los terrenos de entre 250 y $1500 \mathrm{~m}^{2}$ de las mismas zonas.
} 
Ventura, V. Producción y productividad de la normativa urbana. El trabajo político de los sectores medios en sus contiendas por la ciudad. Derecho y Ciencias Sociales. Mayo- Octubre 2019 NN $^{\circ} 1$. (Derecho, ciudad y propiedad) Pgs 82-103. ISNN 1852-2971. Instituto de Cultura Jurídica y Maestría en Sociología Jurídica. FCJ y S. UNLP

En síntesis, el caso muestra cómo los capitales de los sectores medios operan con cierta circularidad: movilizando redes de contactos y experticias profesionales, les beneficiares lograron incidir en la normativa urbana; a su vez, mediante la decisión pública de recualificar suelo o bien se apropiaron de la valorización inducida por la ordenanza (vía 3) o bien lograron un importante ahorro en el precio del $\mathrm{m}^{2}$ (vía 2). Finalmente, además de capital social y cultural (Bourdieu, 1985) les beneficiaries requirieron determinado poder de pago para optar por ambas modalidades, dado que los costos de urbanización de los predios fueron asumidos (en la mayoría de los casos) por elles en carácter de futures propietaries. Por ejemplo, la estructura de costos de los lotes generados por la vía 2 muestra que el 34\% del precio final del lote corresponde a los costos de urbanización (Vértiz, 2016). Es decir, para lograr la subdivisión de los predios les beneficiaries debían contar con 46.989 pesos (de los 139 mil pesos pagados por un lote promedio de $399 \mathrm{~m}^{2}$ ) por fuera del financiamiento del Programa.

\section{Conclusión}

Buscando conocer más sobre cómo se rigen los procesos de producción y apropiación del espacio urbano - especialmente atentos al desempeño de las clases medias- en la primera parte de este artículo nos detuvimos en las prácticas de la población beneficiaria del PROCREAR y en el trabajo político que llevaron adelante para disputar y dar forma a la implementación local del programa nacional.

Indagando en la productividad de sus prácticas, nos interesamos en primer lugar en la presencia de numerosos profesionales en el grupo. Tecnificar y burocratizar ellos mismos sus demandas, es decir sin necesidad de recurrir a profesionales externos al colectivo que realizaran ese trabajo de traducción, les permitió entablar un vínculo ágil con el gobierno municipal. Encontramos además que sus redes interpersonales (amplias y puestas en juego) así como el conocimiento de los "laberintos del Estado", también hicieron posible orientar de un modo particular la implantación local del PROCREAR. Por otra parte, mediante el intercambio de favores "entre iguales" lograron personalizar la política "por mano propia". La política aparece así en su versión instrumental del mismo modo en que lo hace en las redes clientelares de los barrios pobres; la diferencia es que, en nuestro caso, el intercambio de dones se dio entre pares.

Asimismo, sostuvimos que la celeridad con que se canalizó la demanda de la población beneficiaria debe ser leída a contra luz de la espera, una de las formas que asume la dominación política de los sectores populares. En este punto es importante aclarar que la coyuntura del municipio, especialmente aquellos aspectos vinculados a las estrategias de acumulación política del bruerismo, son nodales para comprender este proceso y serán abordados en futuros trabajos. 
Ventura, V. Producción y productividad de la normativa urbana. El trabajo político de los sectores medios en sus contiendas por la ciudad. Derecho y Ciencias Sociales. Mayo- Octubre 2019 NN $^{\circ} 1$. (Derecho, ciudad y propiedad) Pgs 82-103. ISNN 1852-2971. Instituto de Cultura Jurídica y Maestría en Sociología Jurídica. FCJ y S. UNLP

Finalmente repusimos cómo, al intervenir en el contenido de la normativa, les beneficiares incidieron en la producción y apropiación de ciudad, hecho relevante si consideramos que las modalidades que impulsaron fueron las que mayor demanda absorbieron en la ciudad. Así, en la segunda parte del artículo nos detuvimos en exponer la productividad territorial y económica de la ordenanza 11.094/13. Sostuvimos que la normativa, por un lado, reprodujo un modo de producir ciudad donde el mercado fue el principal asignador de recursos y por el otro, promovió la expansión extensa y difusa del perímetro urbano. Mostramos además cómo las rezonificaciones habilitadas por la ordenanza produjeron una fuerte valorización del suelo, la cual en parte fue apropiada por les dueñes de los predios y en parte por les beneficiaries, habilitando una capitalización económica que trascendió al propio acceso a la vivienda.

\section{Bibliografía}

Adamovsky, E. (2013). "Clase media": reflexiones sobre los (malos) usos académicos de una categoría. Revista Nueva Sociedad [en línea] (247). Disponible en: https://nuso.org/articulo/clase-media-reflexiones-sobre-los-malos-usos-academicos-de-unacategoria/

Auyero, J. (2013). Pacientes del estado. Buenos Aires: EUDEBA.

Auyero, J., y Benzecry, C. (2016). La lógica practica del dominio clientelista. Revista Mexicana de Ciencias Políticas y Sociales [en línea], 61 (226), 221-246. Disponible en: http://www.revistas.unam.mx/index.php/rmcpys/article/view/50048/48469

Azuela, A. (2016). Introducción. Una especie de neorrealismo jurídico. En: Azuela, A. (Coord.) La ciudad y sus reglas. Sobre la huella del derecho en el orden urbano (pp. 9-41). Mexico: UNAM, Instituto de Investigaciones Sociales.

Azuela, A., y Mussetta, P. (2009). Algo más que el ambiente. Revista de Ciencias Sociales [en línea] (16), 191-215. Disponible en: http://www.unq.edu.ar/advf/documentos/5940320790edb.pdf

Bourdieu, P. (2007). El sentido práctico. Buenos Aires: Siglo XXI.

Bourdieu, P. (1985). Espacio social y génesis de "las clases". Espacios (2), 27-55.

Canestraro, M. L. (2014). Algunas hipótesis para reflexionar sobre el impacto del Pro.Cre.Ar en la dinámica urbana marplatense. Ponencia presentada en VIII Jornadas de Sociología de la $U N L P$. La Plata. 
Ventura, V. Producción y productividad de la normativa urbana. El trabajo político de los sectores medios en sus contiendas por la ciudad. Derecho y Ciencias Sociales. Mayo- Octubre 2019 NN $^{\circ} 1$. (Derecho, ciudad y propiedad) Pgs 82-103. ISNN 1852-2971. Instituto de Cultura Jurídica y Maestría en Sociología Jurídica. FCJ y S. UNLP

Cosacov, N. (2014). Habitar la centralidad. Trayectorias residenciales y uso del espacio urbano de residentes en Caballito, Buenos Aires. Tesis doctoral, Facultad de Ciencias Sociales, Universidad de Buenos Aires.

Cosacov, N. (2017a). El papel de la familia en la inscripción territorial. Exploraciones a partir de un estudio de hogares de clase media en el barrio de Caballito, Buenos Aires. Población \& Sociedad, 24, 35-65.

Cosacov, N. (2017b). Construyendo un barrio "de clase media". Narrativas, moralidades e identidades de clase media en disputas urbanas en un barrio de Buenos Aires. En: Boy, M. \& Perelman, M. (coords.), Fronteras en la ciudad (re)producción de desigualdades y conflictos urbanos. Buenos Aires: Teseo Press.

Cravino, M. C. (2010). Percepciones de los nuevos espacios urbanos en Buenos Aires. Un analisis del Plan Federal de Viviendas desde la perspectiva de los receptores. Dearq06, 20-31.

Danani, C. (2009). La gestión de la política social: un intento de aportar a su problematización”. En: Chiara, M. y Di Virgilio, M. (Comp.) Manual de Gestión de Política Social. Los Polvorines: UNGS.

Del Río, J. P. (2010). El lugar de la vivienda social en la ciudad. Una mirada desde el mercado de localizaciones intra-urbanas y las trayectorias habitacionales de los destinatarios. Ponencia presentada en III Jornadas del Doctorado en Geografía. Desafios Teóricos y compromiso social en la argentina de hoy. Facultad de Humanidades y Ciencias de la Educación. La Plata.

Del Río, J. P. (2016). Tensiones entre hipoteca, suelo y política urbana. El caso del Pro.Cre.Ar en el partido de La Plata, provincia de Buenos Aires. Estudios socioterritoriales. Revista de Geografía (19), 135-151.

Del Río, J.P (2017). Crédito hipotecario, acceso al suelo y clase media en la implementación del Pro.Cre.Ar en la ciudad de La Plata. En: Cravino, M.C. (Coord.) Detrás de los conflictos. Estudios sobre la desigualdad urbana en la Región Metropolitana de Buenos Aires (pp.265305). Los Polvorines: UNGS.

Di Virgilio, M. M. (2007). Trayectorias residenciales y estrategias habitacionales de sectores medios y populares en Buenos Aires. Tesis doctoral, Facultad de Ciencias Sociales, Universidad de Buenos Aires.

Fava, R. (2010). La clase media, entre la cultura y la historia. Representaciones sobre la clase media en el conflicto entre "vecinos" y "ocupantes" durante la toma del Parque Indoamericano en 2010., inédito. 
Ventura, V. Producción y productividad de la normativa urbana. El trabajo político de los sectores medios en sus contiendas por la ciudad. Derecho y Ciencias Sociales. Mayo- Octubre 2019 № 21. (Derecho, ciudad y propiedad) Pgs 82-103. ISNN 1852-2971. Instituto de Cultura Jurídica y Maestría en Sociología Jurídica. FCJ y S. UNLP

Garguin, E. (2006). La formación histórica de la clase media en Argentina. Una aproximación bibliográfica. Revista Apuntes (11), 228-239.

Jessop, B. (2017). El Estado. Pasado, Presente, Futuro. Madrid: Catarata.

Kessler, G. y Di Virgilio, M. (2005). The new poverty in Argentina and Latino America. En: Hanley, L., Ruble, B. y Tulchin, J. (Comps.) Becoming Global and the New Poverty of Cities. Agencia de los Estados Unidos para el Desarrollo Internacional.

Kessler, G. y Di Virgilio, M. (2008). La nueva pobreza urbana: dinámica global, regional y argentina en las últimas dos décadas. Revista de la CEPAL (95), 31-50.

Latour, B. (1998). De la mediación técnica: filosofía, sociología y genealogía. En Domenech, M. y Tirado, F. J. (Comps.) Sociología asimétrica. Barcelona: Gedisa.

Melé, P. (2006). Pour une géographie de l'action publique. Patrimoine, environnement et processus de territorialisation. Habilitation á diriger des recherches. Université Francois Rabelais, Tours.

Melé, P. (2016). ¿Qué producen los conflictos urbanos?. En: Carrión, F. y Erazo, J. (Edits.) El derecho a la ciudad en America Latina, Visiones desde La Poltica (pp. 127-157). México: PUEC-UNAM.

Olson, M. (1965). The Logic of Collective Action, Cambridge: Harvard University Press.

Ostuni, F. (2010). Políticas nacionales y escenarios locales. Reflexiones sobre la implementacion del Programa Federal de Construcción de Vivienda en la Ciudad de Buenos Aires. Cuadernos de Vivienda y Urbanismo, 3 (5), 12-33.

Pírez, P. (1995). Actores sociales y gestión de la ciudad. Ciudades (28).

Perelmiter, L. (2007). ¿Es posible la debildiad estatal? Papeles de trabajo. Revista electrónica del Instituto de Altas Estudios Sociales de la Universidad Nacional de General San Martín. (2), $1-19$.

Perelmiter, L. (2016). Burocracia plebeya. La trastienda de la asistencia social en el Estado Argentino. San Martín: UNSAM.

Potocko, A. (2015). Apuntes de la implementación de las políticas de vivienda desde los actores. El Programa Federal de Emergencia Habitacional en el barrio 2 de abril en Humahuaca (Jujuy, Argentina) . Cuadernos Urbanos, 111-139.

Rosaldo, R. (1991). Cultura y Verdad. México: Grijalbo.

Ventura, V. (2015). El Pro.Cre.Ar en la ciudad de La Plata: surgimiento y rasgos de la ordenanza 11094/13, la opción municipal ante la falta de suelo urbano. Ponencia presentada en 
Ventura, V. Producción y productividad de la normativa urbana. El trabajo político de los sectores medios en sus contiendas por la ciudad. Derecho y Ciencias Sociales. Mayo- Octubre 2019 NN $^{\circ} 1$. (Derecho, ciudad y propiedad) Pgs 82-103. ISNN 1852-2971. Instituto de Cultura Jurídica y Maestría en Sociología Jurídica. FCJ y S. UNLP

Segundas Jornadas de Sociología de la Facultad de Ciencias Politicas y Sociales de la UNCuyo. Mendoza.

Vértiz, F. (2016). La intervención estatal en la producción de la ciudad. Conformación y orientación de la política urbana en el partido de La Plata (2003-2014). Tesis doctoral. Facultad de Humanidades y Ciencias de la Educación, Universidad Nacional de La Plata. 